\title{
Lung Ultrasound Findings of Patients with Dengue Infection: A Prospective Observational Study
}

\author{
Hiroshi Koyama, ${ }^{1,2,3^{\star}}$ Wirongrong Chierakul, ${ }^{1,4}$ Prakaykaew Charunwatthana, ${ }^{1,4}$ Natpatou Sanguanwongse,${ }^{5}$ Benjaluck Phonrat, ${ }^{1}$ \\ Udomsak Silachamroon, ${ }^{1}$ and Arjen M. Dondorp ${ }^{4,6}$ \\ ${ }^{1}$ Department of Clinical Tropical Medicine, Faculty of Tropical Medicine, Mahidol University, Bangkok, Thailand; ${ }^{2}$ Department of Emergency and \\ Critical Care Medicine, Jichi Medical University, Saitama Medical Center, Saitama, Japan; ${ }^{3}$ Department of Critical Care Medicine, Shonan Kamakura \\ General Hospital, Kanagawa, Japan; ${ }^{4}$ Mahidol-Oxford Research Unit (MORU), Faculty of Tropical Medicine, Mahidol University, Bangkok, Thailand; \\ ${ }^{5}$ Department of Medicine, Bamrasnaradura Infectious Diseases Institute, Nonthaburi, Thailand; ${ }^{6}$ Centre for Tropical Medicine and Global Health, \\ Nuffield Department of Medicine, University of Oxford, Oxford, United Kingdom
}

\begin{abstract}
Lung ultrasound (LUS) is a more sensitive method of detecting pathological pulmonary changes than chest X-ray. Therefore, LUS for patients with dengue could be an important tool for the early detection of pleural effusions and pulmonary edema signifying capillary plasma leakage, which is the hallmark of severe dengue pathophysiology. We conducted a prospective observational study of pulmonary changes identifiable with LUS in dengue patients admitted to the Hospital for Tropical Diseases in Mahidol University, Bangkok, and the Bamrasnaradura Infectious Diseases Institute, Nonthaburi, Thailand. The LUS findings were described according to standard criteria, including the presence of A, B1, B2, and C patterns in eight chest regions and the presence of pleural effusions. From November 2017 to April 2018, 50 patients with dengue were included in the study. LUS was performed during the febrile phase for nine patients (18\%) and during the criticalconvalescence phase for 41 patients (82\%). A total of 33 patients $(66 \%)$ had at least one abnormality discovered using LUS. Abnormal LUS findings were observed more frequently during the critical-convalescence phase $(N=30 / 41 ; 73 \%)$ than during the febrile phase $(N=3 / 9 ; 33 \%)(P=0.047)$. Abnormal aeration patterns were observed in 31 patients $(62 \%)$. Only $B$ patterns with only multiple $B$ lines were observed in 21 patients (42\%); of these patients, three had already exhibited B patterns during the febrile phase $(N=3)$. C patterns $(N=10 ; 24 \%)$, pleural effusion $(N=10 ; 24 \%)$, and subpleural abnormalities $(N=11 ; 27 \%)$ were observed only during the critical-convalescence phase. LUS can detect signs of capillary leakage, including interstitial edema and pleural effusions, early during the course of dengue.
\end{abstract}

\section{INTRODUCTION}

Dengue is a growing public health concern in tropical and subtropical countries. ${ }^{1}$ Capillary leakage with hemoconcentration is central to the pathogenesis of severe dengue, and early recognition is important to inform patient management, including appropriate fluid administration. ${ }^{2}$ Well-established indicators for plasma leakage with severe dengue are pleural effusions and an increased hematocrit level. Increased vascular permeability can also cause pulmonary edema, which is occasionally aggravated by cardiac compromise. ${ }^{3-6}$ Other pulmonary involvement in dengue can be caused by a virus infecting the lung parenchyma with direct cytopathic effects, thus leading to lung infiltrates. ${ }^{7,8}$ Two retrospective studies described infiltrative abnormalities found by the chest X-ray or chest CT independent of the presence of pleural effusion in more than $20 \%$ of patients with dengue hemorrhagic fever (DHF) and dengue shock syndrome (DSS). ${ }^{9,10}$ In clinical practice, chest X-ray is the most common imaging technique for identifying pleural effusions, pulmonary edema, and lung infiltrates in dengue patients. However, lung ultrasound (LUS) might be a more sensitive method of detecting pleural effusions and pulmonary edema, thereby allowing for early recognition of capillary leakage before the beginning of the critical phase of the disease, which usually starts at the moment of defervescence. ${ }^{11}$ In addition, LUS might be a sensitive alternative method for the early detection of other parenchymal pathology in dengue patients.

The utilization of LUS for acutely ill patients has expanded over the past decade. LUS has been shown to be superior to

*Address correspondence to Hiroshi Koyama, Department of Critical Care Medicine, Shonan Kamakura General Hospital, 13701, Okamoto, Kamakura, Kanagawa, 247-8533, Japan. E-mail: ko1978hiro@gmail.com conventional chest $X$-ray for detecting pneumonia, ${ }^{12,13}$ atelectasis, ${ }^{14}$ pneumothorax, ${ }^{15}$ pulmonary edema, ${ }^{16}$ and pleural effusion. ${ }^{17-19}$ Multiple $B$ lines, which are laser-like vertical hyperechoic artifacts arising from the pleural line to a distal end without attenuation, represent increased density cause by the loss of aeration in the lung periphery, which can be caused by pulmonary edema or interstitial lung disease. ${ }^{20} \mathrm{~A}$ previous study showed that multiple $B$ lines were frequently observed among patients with severe dengue and a hemodynamic profile of cardiogenic shock. ${ }^{21}$ However, there is little information regarding the performance of LUS to identify lung parenchymal abnormalities in patients with dengue of varying severity. We hypothesized that LUS for dengue patients can detect early lung pathological abnormalities, particularly those caused by capillary leakage. We conducted an observational study to describe pulmonary pathology detected by LUS in patients with dengue at various stages of the disease. We focused on the early detection of pulmonary edema as an early sign of dengue-associated capillary leakage.

\section{METHODS}

Study design and participants. This prospective observational study was conducted at two hospitals in Thailand (Hospital for Tropical Diseases, Mahidol University in Bangkok, and Bamrasnaradura Infectious Diseases Institute in Nonthaburi). Consecutive admitted patients 15 years or older with fever within 21 days of admission were screened daily for eligibility. Patients with a clinical diagnosis of dengue were asked to participate in the study. Patients who were suspected to have a concomitant acute infection were not included in the study. Written informed consent was obtained from all patients or their relatives before enrollment in the study. The current study was part of a larger study investigating LUS abnormalities 
involved in a range of tropical diseases. The study was approved by the ethics committees from both hospitals (MUTM 2017-072-01).

Data collection. Demographic data, fever duration, and accompanying symptoms were extracted from the medical charts or obtained directly from the patients by the research team. Vital signs, physical examination findings, and relevant laboratory data were also extracted from the medical charts.

Lung ultrasound. LUS was performed for eligible patients soon after consent was obtained. All LUS examinations were performed and interpreted by an experienced LUS examiner (H. K.) who was not involved in patient management. Of the LUS examinations, 10\% was randomly evaluated by an external LUS expert (L. P.). A single ultrasound machine (VIVID-I; General Electric Company, Boston, MA) with a convex probe $(1.3-4.0 \mathrm{MHz})$ was used during the entire study. Patients were examined in the supine or semi-recombinant position covering eight zones of the lung according to the international evidence-based recommendations for point-of-care LUS. ${ }^{20}$ Abnormal findings were registered according to the lung zone and included multiple B lines (three or more B lines per intercostal space), consolidation, subpleural abnormalities, and pleural effusion. Aeration patterns in each scanning zone were classified into four categories: A pattern, B1 pattern, B2 pattern, and $C$ pattern. These categories were defined as follows: A pattern, only $A$ lines or two or fewer $B$ lines on the pleura in an intercostal space; B1 pattern, multiple B lines occupying $50 \%$ or less of the pleura in an intercostal space; B2 pattern, multiple B lines occupying more than $50 \%$ of the pleura in an intercostal space; and C pattern, consolidation with severe or complete aeration loss. Subpleural nodulelike regions and small consolidations were grouped together as subpleural abnormalities because these two entities cannot be clearly differentiated. ${ }^{20}$

Diagnosis of dengue. The provisional diagnosis of dengue was made by a local physician with or without laboratory confirmation according to local guidelines. ${ }^{22,23}$ The diagnosis of dengue was confirmed in the laboratory by either a positive serum dengue NS1 antigen test or a positive dengue IgM antibody test. Seroconversion of dengue IgM or IgG antibodies with paired serum samples also confirmed dengue in the laboratory. Clinically suspected dengue cases that were not confirmed in the laboratory were classified as suspected dengue. Patients were categorized by the pathophysiological phase of the disease: febrile or critical-convalescence. The critical and convalescence phases were grouped as one phase because the time of defervescence $<38^{\circ} \mathrm{C}$ was difficult to estimate for patients admitted without fever. The febrile phase was defined as the period until the day of defervescence, and the critical-convalescence phase was defined as the period starting the day after defervescence (temperature $<38^{\circ} \mathrm{C}$ ). ${ }^{23,24}$

Statistical analysis. Statistical analysis was performed using SPSS statistical software package (ver. 18.0). Normally distributed numerical variables were summarized as mean \pm $\mathrm{SD}$, and groups were compared using Student's $t$ test. Nonnormally distributed numerical variables were summarized as the median and interquartile range (IQR), and groups were compared using the Mann-Whitney $U$ test. Categorical variables were presented as numbers and proportions and compared using Pearson's $\chi^{2}$ test or Fisher's exact test as appropriate. A two-sided $P<0.05$ was considered statistically significant.

\section{RESULTS}

The study recruitment period was from November 2017 to February 2018, for the Hospital for Tropical Diseases, and from March to April 2018 for the Bamrasnaradura Infectious Disease Institute. A total of 136 febrile patients were screened; of those patients, 50 with a provisional diagnosis for dengue were included to the study (Figure 1). Dengue was confirmed in the laboratory for 44 patients; 41 had positive serum NS1 antigen results, 2 only had positive serum dengue IgM antibody results, and 1 exhibited seroconversion in the dengue

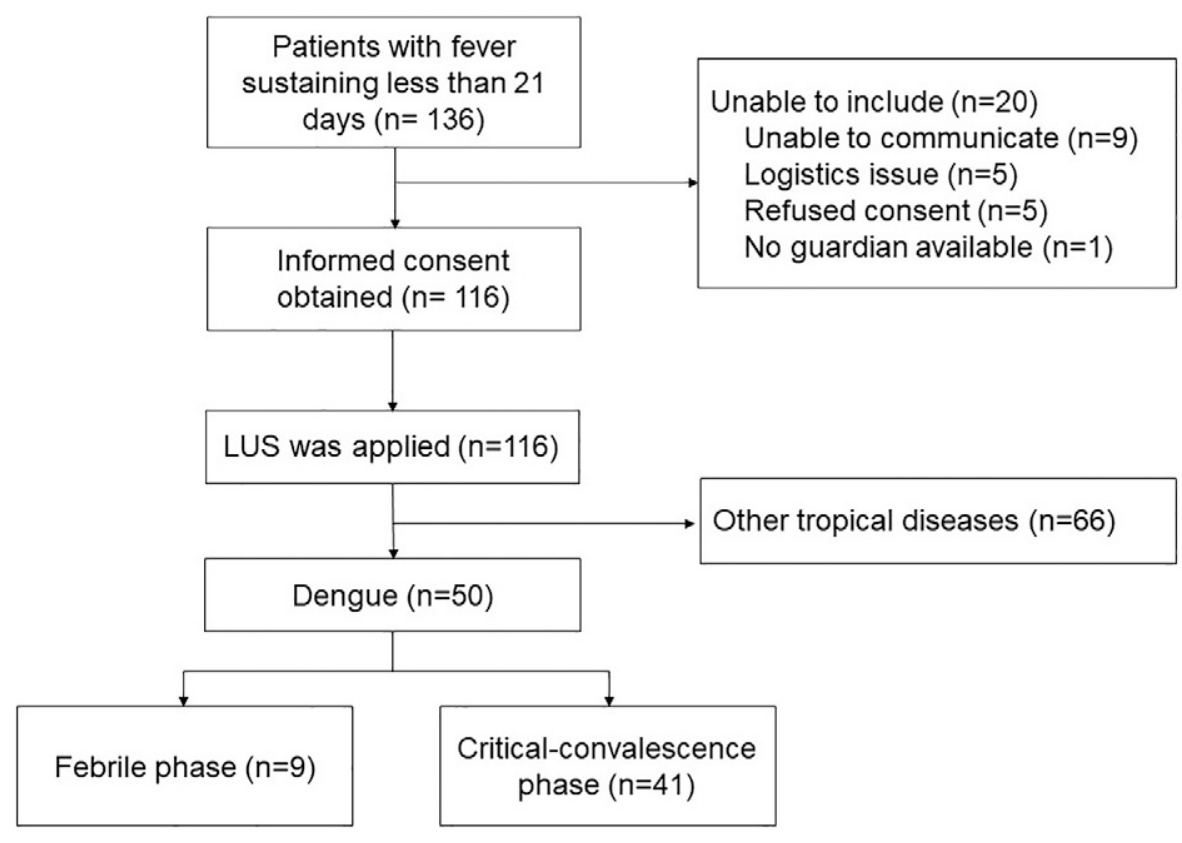

FIGURE 1. Flow diagram of the study. 
IgG antibody titer. The other six patients were classified as having clinically suspected dengue; three patients had negative serum NS1 antigen and dengue IgM antibody results, and three patients did not undergo testing of the serum NS1 antigen or dengue IgM antibody or both. The demographic data, symptoms, and laboratory parameters are summarized in Table 1. No patients had an underlying heart or lung disease that potentially could have affected the results of LUS.

LUS was performed during the febrile phase for nine patients $(18 \%)$ and the critical-convalescence phase for 41 patients $(82 \%)$. Of 50 patients, $33(66 \%)$ had at least one abnormality discovered by LUS. Abnormal LUS findings were grouped according to the disease phase (Table 2). Abnormal LUS findings were observed more frequently during the critical-convalescence phase $(N=30 / 41 ; 73 \%)$ than during the febrile phase $(N=3 / 9 ; 33 \%)(P=0.047)$. Abnormal aeration patterns were observed in 31 patients (62\%). Only $B$ patterns with only B lines were observed in 21 patients (42\%); of these patients, six had bilateral B patterns (12\%) and 15 had unilateral B patterns (30\%) (Figure 2). All C pattern abnormalities $(N=10)$ were detected in the lateral-posterior zones (zone 3/4), and they were only detected during the critical-convalescence phase. Of these, nine coexisted with pleural effusions. B patterns in the anterior zones were observed in three patients who demonstrated $C$ patterns in zone 3/4 during the critical-convalescence phase (Figure 3). Subpleural abnormalities were observed in 11 patients (22.0\%) only during the critical-convalescence phase.
TABLE 2

LUS findings during each clinical phase

\begin{tabular}{llrl}
\hline & Febrile & Critical-convalescence & $P$ value \\
\hline Abnormal LUS findings, $\mathrm{n} \mathrm{( \% )}$ & $3(33.3)$ & $30(73.2)$ & 0.047 \\
All abnormal aeration, $\mathrm{n}(\%)$ & $3(33.3)$ & $28(68.3)$ & 0.067 \\
Only B pattern, $\mathrm{n}(\%)$ & $3(33.3)$ & $18(43.9)$ & 0.716 \\
Bilateral & $1(11.1)$ & $5(12.2)$ & \\
Unilateral & $2(22.2)$ & $13(31.7)$ & \\
C pattern, n (\%) & $0(0)$ & $10(24.4)$ & 0.174 \\
Bilateral & $0(0)$ & $5(12.2)$ & \\
Unilateral & $0(0)$ & $5(12.2)$ & \\
Pleural effusion, n (\%) & $0(0)$ & $10(24.4)$ & 0.174 \\
$\quad$ Bilateral & $0(0)$ & $6(14.6)$ & \\
Unilateral & $0(0)$ & $4(9.8)$ & \\
Subpleural abnormal & $0(0)$ & $11(26.8)$ & 0.177 \\
$\quad$ region, n (\%) & & & \\
& $9(100.0)$ & $41(100.0)$ & \\
\hline
\end{tabular}

\section{DISCUSSION}

This study characterizes lung abnormalities identifiable by LUS during dengue infection. We found that $66 \%$ of patients with nonsevere dengue had abnormal LUS findings. It is important to note that B patterns consistent with early pulmonary edema were observed during all clinical phases of the disease. In the majority of patients, $B$ lines were observed in the absence of pleural effusion. Furthermore, B patterns in the upper lung area were seen concomitant with $C$ patterns (signifying lung consolidation) and pleural effusion in the lower lung

TABLE 1

Patient characteristics

\begin{tabular}{|c|c|c|c|}
\hline & Normal LUS & Abnormal LUS & $P$ value \\
\hline Diagnosis of dengue & & & $0.162 \dagger$ \\
\hline Laboratory-confirmed, n (\%) & $13(76.5)$ & $31(93.9)$ & \\
\hline Clinically suspected, $\mathrm{n}(\%)$ & $4(23.5)$ & $2(6.1)$ & \\
\hline Dengue classification 1997 & & & $0.01^{*}$ \\
\hline Dengue fever, n (\%) & $17(100.0)$ & $20(60.6)$ & \\
\hline Dengue hemorrhagic fever, $\mathrm{n}(\%)$ & $0(0)$ & $13(39.4)$ & \\
\hline Dengue shock syndrome, $\mathrm{n}(\%)$ & $0(0)$ & $0(0)$ & \\
\hline Dengue classification 2009 & & & $0.01^{*}$ \\
\hline Dengue without warning signs, $\mathrm{n}(\%)$ & $11(64.7)$ & $6(18.2)$ & \\
\hline Dengue with warning signs, $\mathrm{n}(\%)$ & $6(35.3)$ & $27(81.8)$ & \\
\hline Severe dengue, $\mathrm{n}(\%)$ & $0(0)$ & $0(0)$ & \\
\hline Age, mean (SD) & $27.0(10.2)$ & $31.3(11.6)$ & 0.2 \\
\hline Sex, male, n (\%)/female, n (\%) & $11(51.5) / 6(48.5)$ & $17(64.7) / 16(35.3)$ & $0.373^{*}$ \\
\hline Total days of fever before admission, median [IQR] & $4.0[2.0]$ & $5.0[2.0]$ & 0.054 \\
\hline Respiratory symptoms, $\mathrm{n}(\%)$ & $6(35.3)$ & $11(33.3)$ & $0.89^{\star}$ \\
\hline Cough, n (\%) & 6 (35.3) & 6 (18.2) & $0.294 \dagger$ \\
\hline Sputum, n (\%) & $4(23.5)$ & 7 (21.2) & $1 \dagger$ \\
\hline Chest pain, $\mathrm{n}(\%)$ & $2(11.8)$ & $3(9.2)$ & $1 \dagger$ \\
\hline Dyspnea, n (\%) & $1(5.9)$ & $2(6.1)$ & $1 \dagger$ \\
\hline \multicolumn{4}{|l|}{ Other symptoms } \\
\hline Malaise, n (\%) & $6(35.3)$ & $24(72.7)$ & $0.01^{*}$ \\
\hline Headache, n (\%) & $13(76.5)$ & $25(75.8)$ & $1 \dagger$ \\
\hline Myalgia, n (\%) & $8(47.1)$ & $24(72.7)$ & $0.073^{*}$ \\
\hline Nausea and vomiting, $\mathrm{n}(\%)$ & $9(52.9)$ & $28(84.8)$ & $0.021+$ \\
\hline Abdominal pain, $\mathrm{n}(\%)$ & $3(17.6)$ & $12(36.4)$ & $0.171^{*}$ \\
\hline Skin rash, $\mathrm{n}(\%)$ & $3(17.6)$ & $8(24.2)$ & $0.728+$ \\
\hline \multicolumn{4}{|l|}{ Laboratory data } \\
\hline Platelet (lowest), ${ }^{*} 10^{3}$ cells $/ \mu \mathrm{L}$, median [IQR] & $41.0[24.5]$ & $24.0[27.5]$ & 0.127 \\
\hline WBC, ${ }^{*} 10^{3}$ cells $/ \mu \mathrm{L}$, median $[\mathrm{IQR}]$ & $3.5[1.9]$ & $3.2[2.2]$ & 0.499 \\
\hline Hematocrit, \%, median [IQR] & $44.0[6.8]$ & $42.9[5.2]$ & 0.774 \\
\hline AST, $\mathrm{U} / \mathrm{L}(N=42)$, median [IQR] & 95.5 [125] & $145.7[149]$ & 0.103 \\
\hline Albumin, $\mathrm{g} / \mathrm{dL},(N=23)$, mean (SD) & $4.1(0.3)$ & $3.9(0.6)$ & 0.431 \\
\hline
\end{tabular}



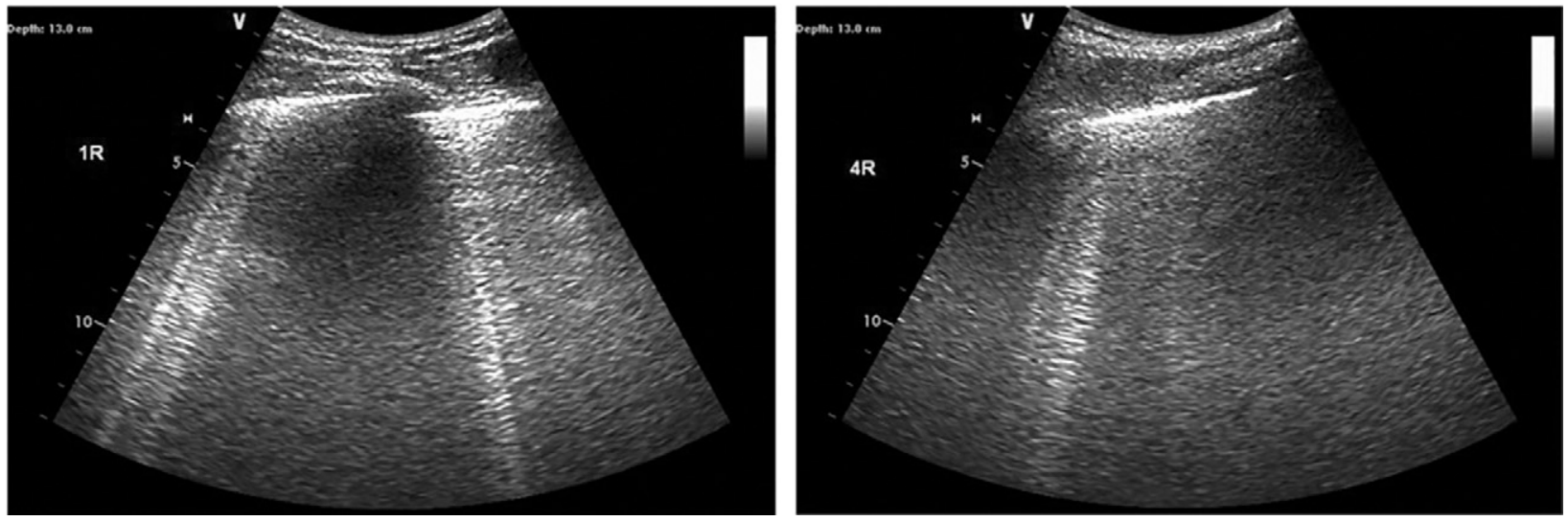

FIGURE 2. Lung ultrasound (LUS) findings in zone $1 \mathrm{R}$ (left) and zone $4 \mathrm{R}$ (right). The LUS was performed during the critical-convalescence phase. B1 and B2 patterns were observed bilaterally without pleural effusion (only B pattern).

area in several patients. $\mathrm{C}$ patterns, pleural effusion, and subpleural abnormalities were observed more frequently after defervescence, whereas B patterns were observed during both the febrile and the critical-convalescence phases.

During this study, $42 \%$ of the patients showed only B patterns, and $33 \%$ of the patients showed $\mathrm{C}$ patterns in the lower lung zone with B patterns in the upper lung zones. Concomitant additional pulmonary conditions, such as interstitial lung diseases or cardiac failure, as alternative causes of observed B patterns, were not clinically probable explanations. The observed $\mathrm{B}$ lines most likely represented subpleural interstitial edema related to pulmonary capillary leakage, which is a hallmark of dengue pathophysiology. ${ }^{16,25}$ LUS is likely a more sensitive method of detecting early subpleural interstitial edema in dengue compared to chest $X$-ray. It has been shown that the sensitivity of chest X-ray for detecting pleural effusion is lower than that of LUS, ${ }^{17-19}$ and small pleural effusions are easily overlooked on chest X-ray. Rodrigues et al. retrospectively investigated chest CT images of 29 patients ( 9 with dengue fever and 20 with dengue with warning signs or severe dengue) out of 2020 dengue patients. ${ }^{10}$ Among 17 patients with abnormal chest CT findings (5 with dengue fever and 12 with dengue with warning signs or severe dengue), 11 patients had abnormal findings in the lung parenchyma and 16 patients had pleural effusions. In the lung parenchyma, ground-glass opacities were the most common finding, followed by consolidation, air space nodules, interlobar septal thickening, and peribronchovascular interstitial thickening. Of these, the subpleural radiographical abnormalities were likely exhibited as B patterns on LUS. ${ }^{26}$

The $\mathrm{C}$ patterns were observed in $20 \%$ of patients, and only during the critical-convalescence phase. Almost all patients with $\mathrm{C}$ pattern abnormalities also had pleural effusions. Although both pneumonia and atelectasis can present as $\mathrm{C}$ patterns on LUS, ${ }^{20}$ the $\mathrm{C}$ patterns in our study more likely represented absorption atelectasis because the $\mathrm{C}$ pattern was only observed at the lung base without a dynamic air bronchogram. ${ }^{27}$ Consolidation on chest CT images has been observed for dengue patients ${ }^{10}$; lung infiltration caused by parenchymal damage related to the direct cytopathic effects of the virus might be another possible cause of the observed $C$ pattern on LUS. Subpleural abnormalities, including subpleural small consolidation and nodule-like regions, were observed only during the critical-convalescence phase. These abnormalities were not just observed at the lung base; hence, these might represent plasma leakage, which is also responsible for the multiple B lines on LUS, as observed during this study.

There were several limitations to our study. First, we could not compare our LUS findings with those of chest X-ray or chest CT for the same patients because these were not available. Second, it was impossible to obtain serial LUS sessions for the same patient during the hospital stay because of
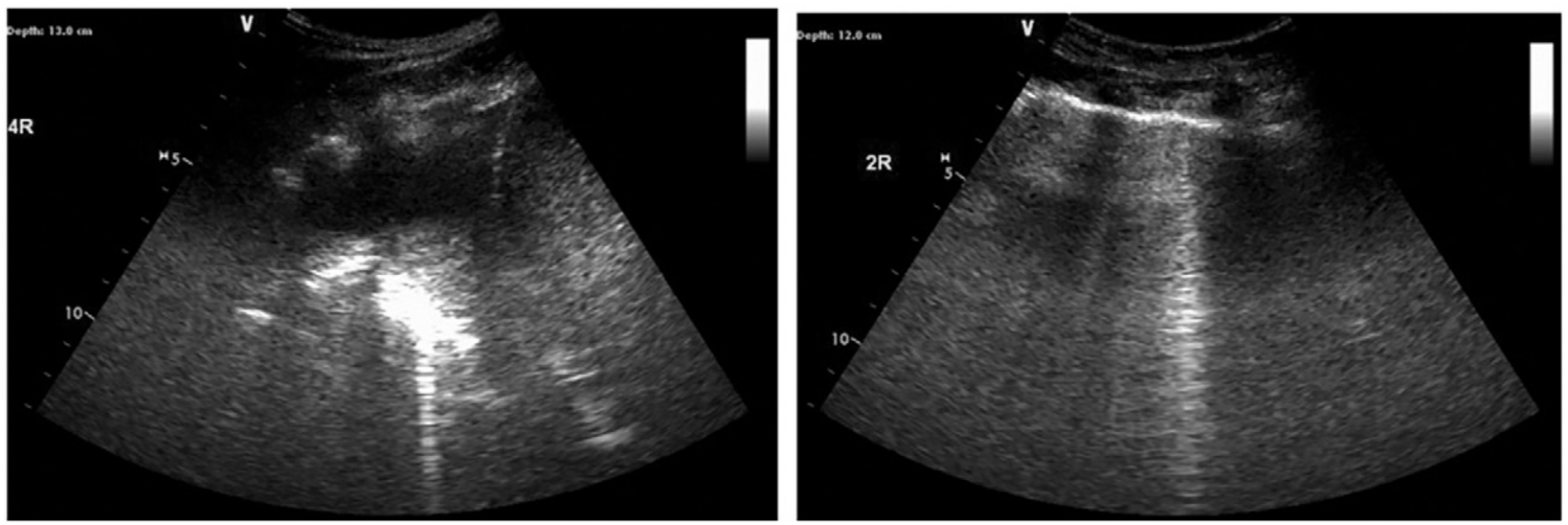

FIGURE 3. Lung ultrasound (LUS) findings in zone 4R (left) and zone 2R (right). The LUS was performed during the critical-convalescence phase. The bilateral $\mathrm{C}$ pattern with pleural effusion was observed in the lateral-posterior zones. The bilateral B2 pattern was observed in the anterior zones. 
logistical constraints, which limited the ability to describe in more detail the relationship over time between $B$ line evolution and pleural effusions. Third, the majority of patients were admitted to the hospital just before or after defervescence. Therefore, febrile patients were underrepresented in this study. Fourth, we could not include patients with hemodynamic shock. However, we could demonstrate that abnormal LUS patterns exist even with nonsevere dengue infections. Fifth, dengue was not laboratory-confirmed for some of the enrolled patients. However, the clinical course of these patients was compatible with dengue, and the LUS findings for this group of patients were not essentially different from those of patients with confirmed dengue.

In conclusion, this study describes pulmonary changes identified by LUS in dengue patients at different clinical stages of the disease. B lines, representing capillary leakage, were observed during both clinical phases, and even with nonsevere dengue infection. Future studies should focus on the ability of LUS findings to predict the progression to severe plasma leakage, which is the hallmark of severe dengue.

Received September 29, 2020. Accepted for publication May 27, 2021. Published online July 19, 2021.

Acknowledgments: We thank all the staff members at the Hospital for Tropical Diseases, Faculty of Tropical Medicine, at Mahidol University for the research. We also thank Piyawadee Chathaisong and the medical staff members at the Bamrasnaradura Infectious Diseases Institute for their support collecting the data. We express sincere appreciation to Prof. Marcus J Schultz, Luigi Pisani, Stije Leopold, and Haruhiko Ishioka, who provided implications for the research.

Financial support: This work was funded by Faculty of Tropical Medicine, Mahidol University.

Authors' addresses: Hiroshi Koyama, Department of Critical Care Medicine, Shonan Kamakura General Hospital, Kanagawa, Japan, E-mail: ko1978hiro@gmail.com. Wirongrong Chierakul, Prakaykaew Charunwatthana, Benjaluck Phonrat, and Udomsak Silachamroon, Department of Clinical Tropical Medicine, Faculty of Tropical Medicine, Mahidol University, Bangkok, Thailand, E-mails: wirongrong.chi@mahidol.ac.th, prakaykaew.cha@mahidol.ac.th, benjaluck.pho@mahidol.ac.th, and udomsak.sil@mahidol.ac.th. Natpatou Sanguanwongse, Department of Medicine, Bamrasnaradura Infectious Disease Institute, Nonthaburi, Thailand, E-mail: natpatou@yahoo.com. Arjen M. Dondorp, Mahidol-Oxford Tropical Medicine Research Unit (MORU), Faculty of Tropical Medicine, Mahidol University, Bangkok, Thailand, E-mail: arjen@ tropmedres.ac.

\section{REFERENCES}

1. Wilder-Smith A, Ooi E-E, Horstick O, Wills B, 2019. Dengue. Lancet 393: 350-363.

2. Taylor WR et al., 2015. Dengue in adults admitted to a referral hospital in Hanoi, Vietnam. Am J Trop Med Hyg 92: 1141-1149.

3. de Almeida RR, Paim B, de Oliveira SA, Souza AS, Gomes ACP, Escuissato DL, Zanetti G, Marchiori E, 2017. Dengue hemorrhagic fever: a state-of-the-art review focused in pulmonary involvement. Lung 195: 389-395.

4. Miranda $\mathrm{CH}$ et al., 2013. Evaluation of cardiac involvement during dengue viral infection. Clin Infect Dis 57: 812-819.

5. Yacoub S, Griffiths A, Hong Chau TT, Simmons CP, Wills B, Hien TT, Henein M, Farrar J, 2012. Cardiac function in Vietnamese patients with different dengue severity grades. Crit Care Med 40: 477-483.

6. Kirawittaya T, Yoon I, Wichit S, Green S, Ennis FA, Gibbons RV, Thomas SJ, Rothman AL, Kalayanarooj S, Srikiatkhachorn A, 2015. Evaluation of cardiac involvement in children with dengue by serial echocardiographic studies. PLoS Negl Trop Dis 9: e0003943.

7. Jessie K, Fong MY, Devi S, Lam SK, Wong KT, 2004. Localization of dengue virus in naturally infected human tissues, by immunohistochemistry and in situ hybridization. J Infect Dis 189: 1411-1418.

8. Póvoa TF, Alves AMB, Oliveira CAB, Nuovo GJ, Chagas VLA, Paes MV, 2014. The pathology of severe dengue in multiple organs of human fatal cases: histopathology, ultrastructure and virus replication. PLoS One 9: e83386.

9. Wang CC, Wu CC, Liu JW, Lin AS, Liu SF, Chung YH, Su MC, Lee IK, Lin MC, 2007. Chest radiographic presentation in patients with dengue hemorrhagic fever. Am J Trop Med Hyg 77: 291-296.

10. Rodrigues RS, Brum ALG, Paes MV, Povoa TF, Basilio de Oliveira CA, Marchiori E, Borghi DP, Ramos GV, Bozza FA, 2014. Lung in dengue: computed tomography findings. PLoS One 9: e96313.

11. Srikiatkhachorn A et al., 2007. Natural history of plasma leakage in dengue hemorrhagic fever: a serial ultrasonographic study. Pediatr Infect Dis J 26: 283-290.

12. Llamas-Álvarez AM, Tenza-Lozano EM, Latour-Pérez J, 2017. Accuracy of lung ultrasound in the diagnosis of pneumonia in adults: systematic review and meta-analysis. Chest 151: 374 382.

13. Pereda M, Chavez M, Hooper-Miele C, Gilman R, Steinhoff M, Ellington L, Gross M, Price C, Tielsch J, Checkley W, 2015. Lung ultrasound for the diagnosis of pneumonia in children: a meta-analysis. Pediatrics 135: 714-722.

14. Wu HD, Yang PC, Kuo SH, Luh KT, 1989. Ultrasonography in complete chest $\mathrm{X}$-ray opacification of hemithorax. Taiwan $\mathrm{Yi}$ Xue Hui Za Zhi 88: 694-699.

15. Alrajab S, Youssef AM, Akkus NI, Caldito G, 2013. Pleural ultrasonography versus chest radiography for the diagnosis of pneumothorax: review of the literature and meta-analysis. Crit Care 17: R208.

16. Pivetta $E$ et al., 2015. Lung ultrasound-implemented diagnosis of acute decompensated heart failure in the ED: a SIMEU multicenter study. Chest 148: 202-210.

17. Gryminski J, Krakówka P, Lypacewicz G, 1976. The diagnosis of pleural effusion by ultrasonic and radiologic techniques. Chest 70: 33-37.

18. Soni NJ, Franco R, Velez MI, Schnobrich D, Dancel R, Restrepo MI, Mayo PH, 2015. Ultrasound in the diagnosis and management of pleural effusions. J Hosp Med 10: 811-816.

19. Emamian SA, Kaasbol MA, Olsen JF, Pedersen JF, 1997. Accuracy of the diagnosis of pleural effusion on supine chest $X$ ray. Eur Radiol 7: 57-60.

20. Volpicelli $\mathrm{G}$ et al., 2012. International evidence-based recommendations for point-of-care lung ultrasound. Intensive Care Med 38: $577-591$

21. Thanachartwet $V$, Wattanathum $A$, Sahassananda D, Wacharasint $P$, Chamnanchanunt S, Khine Kyaw E, Jittmittraphap A, Naksomphun M, Surabotsophon M, Desakorn V, 2016. Dynamic measurement of hemodynamic parameters and cardiac preload in adults with dengue: a prospective observational study. PLoS One 11: 1-17.

22. WHO, 1997. Dengue Haemorrhagic Fever: Diagnosis, Treatment, Prevention and Control. 2nd edition. Geneva, Switzerland: World Health Organization. Available at: https://apps.who.int/ iris/handle/10665/41988.

23. WHO, 2009. Dengue: Guidelines for Diagnosis, Treatment, Prevention, and Control. Geneva, Switzerland: World Health Organization. Available at: https://apps.who.int/iris/handle/10665/ 44188.

24. WHO, 2012. Handbook for Clinical Management of Dengue. Geneva, Switzerland: World Health Organization. Available at: https://www.who.int/denguecontrol/9789241504713/en/.

25. Martindale JL, Noble VE, Liteplo A, 2013. Diagnosing pulmonary edema. Eur J Emerg Med 20: 356-360.

26. Dietrich CF, Mathis G, Blaivas M, Volpicelli G, Seibel A, Wastl D, Atkinson NSS, Cui XW, Fan M, Yi D, 2016. Lung B-line artefacts and their use. J Thorac Dis 8: 1356-1365.

27. Lichtenstein D, Mezière G, Seitz J, 2009. The dynamic air bronchogram: a lung ultrasound sign of alveolar consolidation ruling out atelectasis. Chest 135: 1421-1425. 